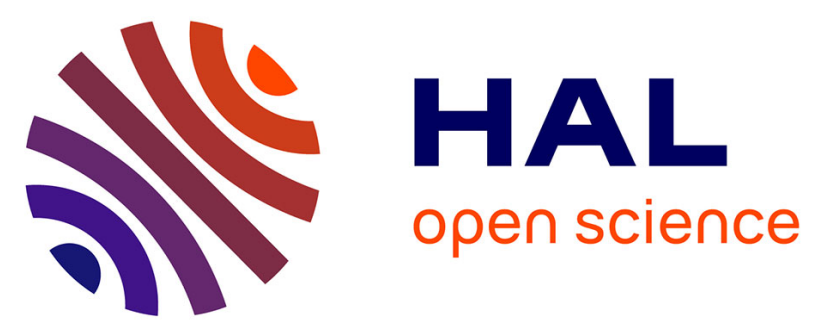

\title{
Building Maps of Local Apparent Conductivity of the Epicardium with a 2D Electrophysiological Model of the Heart
}

\author{
Valérie Moreau-Villéger, Hervé Delingette, Maxime Sermesant, Hiroshi
}

Ashikaga, Elliot R. Mcveigh, Nicholas Ayache

\section{To cite this version:}

Valérie Moreau-Villéger, Hervé Delingette, Maxime Sermesant, Hiroshi Ashikaga, Elliot R. Mcveigh, et al.. Building Maps of Local Apparent Conductivity of the Epicardium with a 2D Electrophysiological Model of the Heart. IEEE Transactions on Biomedical Engineering, 2006, 53 (8), pp.1457-1466. 10.1109/TBME.2006.877794 . inria-00614992

\section{HAL Id: inria-00614992 \\ https://hal.inria.fr/inria-00614992}

Submitted on 17 Aug 2011

HAL is a multi-disciplinary open access archive for the deposit and dissemination of scientific research documents, whether they are published or not. The documents may come from teaching and research institutions in France or abroad, or from public or private research centers.
L'archive ouverte pluridisciplinaire HAL, est destinée au dépôt et à la diffusion de documents scientifiques de niveau recherche, publiés ou non, émanant des établissements d'enseignement et de recherche français ou étrangers, des laboratoires publics ou privés. 


\title{
Building Maps of Local Apparent Conductivity of the Epicardium with a 2-D Electrophysiological Model of the Heart
}

\author{
Valérie Moreau-Villéger, Hervé Delingette, Maxime Sermesant, Hiroshi Ashikaga, \\ Elliot McVeigh and Nicholas Ayache
}

\begin{abstract}
In this article we address the problem of estimating the parameters of an electrophysiological model of the heart from a set of electrical recordings. The chosen model is the reaction-diffusion model on the transmembrane potential proposed by Aliev and Panfilov. For this model, we estimate a local apparent 2D conductivity from a measured depolarization time distribution. First, we perform an initial adjustment including the choice of initial conditions and of a set of global parameters. We then propose a local estimation by minimizing the quadratic error between the depolarization time computed by the model and the measures. As a first step we address the problem on the epicardial surface in the case of an isotropic version of the Aliev and Panfilov model. The minimization is performed using Brent method without computing the derivative of the error. The feasibility of the approach is demonstrated on synthetic electrophysiological measurements. A proof of concept is obtained on real electrophysiological measures of normal and infarcted canine hearts.
\end{abstract}

Keywords: heart modeling, electrophysiology, inverse problem, data assimilation, parameter estimation, reaction-diffusion system

V. Moreau-Villéger, H. Delingette and N. Ayache are with the EPIDAURE research project, INRIA Sophia Antipolis, France

M. Sermesant is with the Computation Imaging Science Group, King's College, London, England

H. Ashikaga and E. McVeigh are with the Laboratory of Cardiac Energetics, National Heart Lung and Blood Institute, National Institute of Health, Bethesda, Maryland, USA 


\section{INTRODUCTION}

\section{A. Motivations}

Cardiac arrhythmias are the cause of considerable morbidity. Tachyarrhytmias can originate from ectopic foci of electrical depolarization or from abnormal conduction pathways in the myocardium. The treatment of choice for patients with tachyarrhythmias is radio-frequency ablation, where the abnormal electrical focus or pathway is ablated by applying radio-frequency energy. For patients with ventricular asynchrony, the treatment of choice is biventricular pacing through a pacing device. An electrophysiological study is performed prior to these interventions: an electrical measurement catheter is inserted into the appropriate chamber of the heart and the electrical activity of the endocardial surface is measured. When inspecting electrophysiological data, cardiologists often base their analysis on the depolarization and repolarization maps of the epicardium or endocardium and more specifically on isochrones associated with both instants. From those maps, expert eyes can detect different electrophysiological pathologies ranging from the existence of low conduction zones caused by infarcted tissue, to the occurrence of fibrillation caused by scrolling waves.

The aim of the research effort presented in this paper is to provide cardiologists with a quantitative and objective map of one or several parameters related to conduction pathologies for a better diagnosis and a better planning of therapies (early detection of damaged cardiac tissue, or a precise location of ectopic foci or reentry points leading to an optimal planning of radiofrequency ablation). To achieve this task we do not resort to a pure signal processing approach, where for instance conduction could be estimated from the distance between two isochrone curves. Instead, we propose to compute this additional information by solving an inverse problem: finding the parameters of a cardiac electrophysiology model that can best explain electrophysiological observations (depolarization time). In this article, we present a first step toward this objective: a method to estimate a local apparent conductivity that will be defined in Section II-C with a 2-D electrophysiological model of the heart from surfacic electrophysiological measures. This work is a first step towards a 3-D study.

\section{B. Context}

In electrophysiology, there are usually three different types of electrical potentials that can be considered: the extracellular potential, the intracellular potential and the transmembrane potential. The cardiac cells are separated from their environment by a membrane, creating two 
distinct electrical spaces, respectively the intra and the extracellular domains which have different ionic concentrations and therefore different potentials, the intra and the extracellular potentials. This difference creates a transmembrane potential (TMP). When a cardiac cell receives a pacing signal, the modification of the transmembrane potential creates an action potential leading to the contraction of cardiac fibers. For this reason, the measurement and computation of the transmembrane potential is crucial to assess the cardiac function.

The state of the art in measuring the electrical activity of the heart is mainly electro-anatomical mapping [1], catheter based measures like the EnSite system (Endocardial Solutions) or contact electrodes on epicardium or endocardium such as the studies presented in [2], [3], [4]. In this paper, the measures were performed using an epicardial sock on several dog hearts. This procedure is very invasive but the electrodes are very accurately located and we show results on normal and infarcted hearts. The infarcted regions have different conduction patterns and therefore we expect the parameters of the model to be different in these regions.

A wide range of models of the electrical activity of the heart [5], [6], [7], [8], [9] has been developed from very accurate cellular models like Luo and Rudy models [10], [11] to phenomenologic efficient models [12], [13], [14], [15]. Accurate models such as bidomain models or Luo-Rudy models provide excellent insight into the physiological phenomena creating the electrical activity of the heart [16] but are probably too sophisticated for our inverse problem. Actually, these models are designed to capture very subtle modifications in the shape of the transmembrane potential [17] whereas we only measure here a depolarization time distribution. Moreover subtle models contain too many parameters to estimate with respect to the number of available measures. The FitzHughNagumo model [12] fits our expectations and allows to perform reasonably fast computations of the transmembrane potential propagation. Aliev and Panfilov have developed a modified version of the FitzHugh-Nagumo equations suited to the cardiac transmembrane potential [18].

In order to compare the transmembrane potential (TMP) computed by the model with the measured extracellular potential, we consider depolarization time. The depolarization time is the moment when a cardiac cell gets activated, leading to fiber contraction. The repolarization time is the moment when a cardiac cell returns to its rest potential, leading to fiber relaxation. The depolarization time can be computed from the extracellular potential measures, as explained in [19], by taking the instant when the time derivative is the most negative (Figure 1). From the transmembrane potential point of view, the depolarization time at a given point is the first instant when the transmembrane potential is above a threshold (Figure 1). As it is rather tedious to extract 


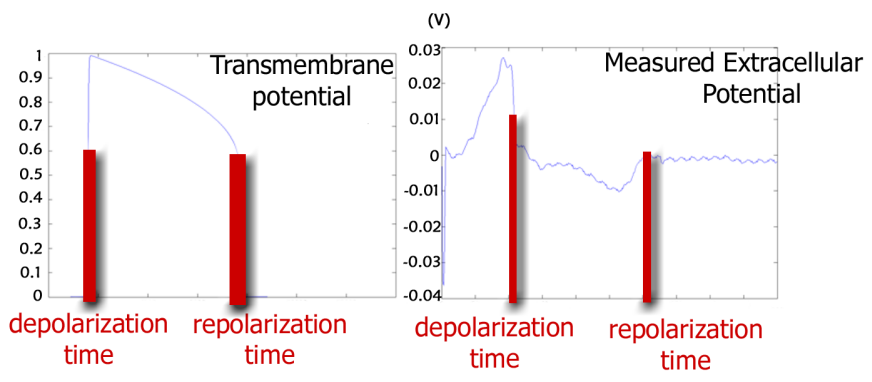

Fig. 1. Comparing simulated transmembrane potential (left) and measured extracellular potential (right) on one electrode.

repolarisation time from measured data, we based our study on the depolarization time only.

Estimating parameters from patient specific data can be addressed as a data assimilation problem. Data assimilation methods [20], [21] improve dynamical models by combining them with real observations. This combination is done via an appropriate minimization of the difference between observations and simulated data. Data assimilation techniques are very popular in meteorology and oceanography. They were recently introduced in biomechanical studies of the heart as in [22] where an extended Kalman filter is used to estimate mechanical parameters of the heart from a real heart motion. None of the classical methods of minimization used in data assimilation are truly suited for the model and the measures of our problem, we thus propose a specific minimization method.

In Section II, we first describe the methods of this study. First we detail the model and the details of the simulation of the electrical wave based on this model. We then specify initial adjustment including the choice of initial conditions and of a set of global parameters before detailing the local apparent conductivity estimation. In Section III, we present the results of this procedure. We first validate the global adjustment and the local estimation on simulated measures. We then show the results of the estimation on a normal and on an infarcted heart. In Section IV, we discuss these results. Finally in Section V, we summarize and present the perspectives of this work.

\section{Methods}

The estimation method that we propose is based on the comparison between simulated and measured depolarization time on the epicardial surface. In this section we first present the Aliev-Panfilov model, followed by a procedure to get initial conditions and a set of parameters that produce a simulation that globally fits the measures. Finally we present a local parameter 
estimation algorithm. This local estimation is performed using Brent method to minimize the quadratic error between measured and simulated depolarization time.

\section{A. Model description}

We chose the following formulation for the model of Aliev and Panfilov.

$$
\begin{aligned}
\varepsilon \partial_{t} u & =\varepsilon^{2} \operatorname{div}(D \nabla(u))+k u(1-u)(u-a)-u z \\
\partial_{t} z & =-(k u(u-a-1)+z)
\end{aligned}
$$

In this formulation, $u$ is a normalized transmembrane potential (between 0 and 1 ) and $z$ is a variable modeling the repolarization, $k$ controls the repolarization, $\varepsilon$ controls the coupling between the transmembrane potential and the repolarization variable $z$ and $a$ controls the reaction phenomenon. A 3D anisotropic model based on the Aliev-Panfilov system was developed in the context of the $\mathrm{ICEMA}^{1}$ collaborative research action [23], [24], [25], [19].

The electrophysiological measures are usually available on the endocardium or the epicardium. A first essential stage before addressing the 3D problem is to tackle a simplified and tractable problem by considering a surface model. Thus we perform a simulation based upon the Aliev and Panfilov model on a surface triangulation $\mathcal{S}$ with a set $\mathcal{V}$ of $N$ vertices and a set $\mathcal{T}$ of $L$ triangles. The surface meshes used in this study are built from the locations of the electrodes of an epicardial sock.

We use a further simplification by considering an isotropic propagation i.e. $D=d \operatorname{diag}(1,1,1)$ in system (1), where the diffusion coefficient $d$ is proportional to a conductivity. The system (1) is scaled spatially to the maximum dimension of the triangulated mesh and temporally so that the action potential duration is around $0.3 \mathrm{~s}(\tau=0.26 t$, with $\tau$ the real time and $t$ the normalized time). The temporal integration of the system (1) is done with an explicit Euler scheme. The spatial integration is performed with the finite elements method with linear triangular elements. The numerical issues and the implementation are described in [26]. Once a simulation of the entire cardiac cycle is performed, based on the Aliev-Panfilov model, the depolarization time distribution is computed using the following equation:

$$
t^{s}=\min \{t \mid u(t)=0.5\}
$$

\footnotetext{
${ }^{1}$ ICEMA/ICEMA-2 : http://www-rocq.inria.fr/sosso/icema2/icema2.html
} 


\section{B. Global adjustment of the model}

This global adjustment of the model includes the adjustment of initial conditions and the choice of a set of values for parameters $a, k, \varepsilon$ and $d$ to scale the propagation in the time and space. The propagation of the depolarization wave is very sensitive to the location of the pacing region. We choose the pacing region by selecting the vertices with the smallest depolarization time.

The parameter $\varepsilon$ is chosen according to the grid size. The choice $\varepsilon=0.1$ guarantees a stable scheme even for very coarse meshes associated to the epicardial sock. The parameters of the model $a, k$, or $d$ can vary between individuals or species. With the knowledge of the depolarization time, we can only adjust one parameter. We choose in a first stage to estimate a global value for the parameter $k$ from the depolarization time while standard values are assigned to the other parameters $(a=0.15$ and $d=1.0)$. The diffusion coefficient $d$ is locally estimated in a second stage (Section II-C).

As stated by [27], the velocity of the depolarization wave on a $1 \mathrm{D}$ domain can be expressed as follows.

$$
c=\sqrt{2 k d}(0.5-a)
$$

In $2 \mathrm{D}$, the velocity of the depolarization wave is not constant in space. At each point in the mesh, it is equal to the velocity in 1D (Equation (3)) minus a term proportional to the curvature of the front [27]. As we only need a global estimate of the propagation velocity on a surface, we neglect, as a first approximation, the front curvature and simply approximate the velocity $c$ of the depolarization wave by its expression in Equation (3).

The depolarization velocity $c$ can also be estimated from the gradient of the measured depolarization time on the surface, $\nabla_{x} t: 1 / c=\left\|\nabla_{\mathbf{x}} t\right\|$. The gradient $\boldsymbol{\nabla}_{x} t$ on the surface can be computed as described in Appendix. Then, we compute a median value of the gradient $\nabla_{x} t$ over the whole mesh and using Equation (3) we get the following approximation: median $\left(\left\|\nabla_{\mathbf{x}} t\right\|\right)^{-1} \approx \sqrt{2 k d}(0.5-a)$.

From this equation, if all parameters but $k$ are fixed, it is possible to compute $k$ directly:

$$
k=\frac{1}{2 d}\left(\frac{\operatorname{median}\left(\left\|\boldsymbol{\nabla}_{\mathbf{x}} t\right\|\right)^{-1}}{0.5-a}\right)^{2}
$$

But by doing so, we compare a theoretical $1 \mathrm{D}$ velocity and an apparent velocity computed on a $2 \mathrm{D}$ surface and this leads to a biased estimation. Therefore, we also compute a velocity estimated from a first simulation on the same mesh as the one used for the measures. As the velocity $c$ is proportional to $1 / \sqrt{k}$, a ratio between measured $c^{m}$ and simulated $c^{s}$ propagation velocity can be 
computed as follows.

$$
\frac{\operatorname{median}\left\|\boldsymbol{\nabla}_{\mathbf{x}} t^{m}\right\|}{\operatorname{median}\left\|\boldsymbol{\nabla}_{\mathbf{x}} t^{s}\right\|}=\frac{c^{s}}{c^{m}} \approx \frac{\sqrt{k^{s}}}{\sqrt{k^{m}}}
$$

The measured and simulated depolarization time distributions are denoted by $t^{m}$ and $t^{s}$ respectively. $k^{s}$ is the initial value for the parameter $k$ in the initial simulation and $k^{m}$ is the new adjusted value. $k^{m}$ can be computed as follows.

$$
k^{m}=k^{s}\left(\frac{\operatorname{median}\left\|\boldsymbol{\nabla}_{\mathbf{x}} t^{s}\right\|}{\operatorname{median}\left\|\boldsymbol{\nabla}_{\mathbf{x}} t^{m}\right\|}\right)^{2}
$$

With this new value for the parameter $k$, we get a complete set of global parameters that leads to a new simulation based on the model. The result of this simulation is the initialization of the local estimation that is described in the next section.

\section{Local estimation of apparent conductivity}

Once the simulated depolarization time map globally fits the measured one, a local adjustment of the model is possible. Depolarization time is an implicit function of the action potential whereas classical data assimilation methods generally require a linear function or at least an explicit function of the results of the model between the observations and the state variables. For that reason, we propose a specific estimation algorithm.

In the case of an infarct, all parameters $a, k$ and $d$ are modified in the infarcted region. The depolarization time distribution depends on the product $\sqrt{k d}(0.5-a)$ and thus only allows to estimate one parameter. We choose the diffusion coefficient $d$ as the spatially varying parameter. Ischemic or infarcted regions exhibit a decrease in electrical conduction so that the velocity of the depolarization wave is smaller in this region and therefore should have a lower diffusion coefficient $d$. Due to the fact that parameters $a$ and $k$ also vary in ischemic regions, the diffusion coefficient $d$ also reflects the composite variations of parameters $k$ and $a$. With the global adjustment of $k$ proposed in section II-B, the diffusion coefficient $d$ can no longer be related to the electrical conductivity. Therefore, we call $d$ the apparent conductivity (AC).

In the discretized model [26], an $\mathrm{AC}$ value is assigned to each triangle. Consequently, we look for an AC map $(\mathbf{d})=\left(d_{j}\right)_{0 \leq j \leq L-1}$, where $L$ is the number of triangles in the triangulation. This AC map should minimize $\mathcal{C}(\mathbf{d})=\sum_{v \in \mathcal{V}}\left(t_{v}^{m}-t_{v}\left(d_{0}, \ldots, d_{L-1}\right)\right)^{2}$ where $\mathcal{V}$ is the set of the vertices in the triangulation, $t_{v}^{m}$ is the measured depolarization time at vertex $v$ and $t_{v}\left(d_{0}, \ldots, d_{L-1}\right)$ the depolarization time at vertex $v$ resulting from a simulation with the conductivities $\left(d_{0}, \ldots, d_{L-1}\right)$. 
In order to have a robust estimate of the $\mathrm{AC}$, we split the heart surface into different connected regions and estimate one $\mathrm{AC}$ value $d_{R}$ for each region $R$. Let $\left(R_{k}\right)_{0 \leq k \leq K-1}$ be a partition of the surface in $K$ regions. For each region $R_{k}, d_{j}=d_{R_{k}}$ for all $j$ such that the $j^{\text {th }}$ triangle of the surface belongs to $R_{k}$. Then, the new minimization problem is to find $(\mathbf{d})=\left(d_{R_{k}}\right)_{0 \leq k \leq K-1}$ that minimizes $\mathcal{C}(\mathbf{d})=\sum_{v \in \mathcal{V}}\left(t_{v}^{m}-t_{v}\left(d_{R_{0}}, \ldots, d_{R_{K-1}}\right)\right)^{2}$

In order to simplify the minimization of $\mathcal{C}$, we want to use the causality of the electrical wave propagation to estimate the $\mathrm{AC}$ one region after another, following the order of depolarization. To check this assumption, we studied the influence of the apparent conductivity $d_{R}$ of one region $R$ on the depolarization time distribution by computing a rough estimation of $\partial t / \partial d_{R}$ based on centered finite differences. For each vertex $v \in \mathcal{V}$,

$$
\frac{\partial t_{v}}{\partial d_{R}} \approx \frac{t_{v}\left(d_{R}+\Delta d\right)-t_{v}\left(d_{R}-\Delta d\right)}{2 \Delta d} .
$$

In order to compute $t_{v}\left(d_{R}+\Delta d\right)$ and $t_{v}\left(d_{R}-\Delta d\right)$, we have to set the $\mathrm{AC}$ of region $R$ to $d_{R}+\Delta d$ and then to $d_{R}-\Delta d$ and perform one simulation for each of these values. We noticed that, as expected, the $\mathrm{AC} d_{R}$ mainly influences regions having depolarization time equal to or greater than the depolarization time of region $R$.

As a consequence, we transform a K-dimensional minimization problem to $K$ successive one dimensional minimization problems:

$$
\mathcal{C}\left(d_{R}\right)=\sum_{v \in \mathcal{V}}\left(t_{v}^{m}-t_{v}\left(d_{R}\right)\right)^{2}
$$

During the estimation of the $\mathrm{AC}$ value $d_{R}$, the conductivities of other regions remain constant.

As there are enough vertices in a region to provide a robust estimate, we simplify the criterion $\mathcal{C}\left(d_{R}\right)$ by taking into account only the vertices of the region $R$ :

$$
\mathcal{C}\left(d_{R}\right)=\sum_{v \in \mathcal{V}, v \in R}\left(t_{v}^{m}-t_{v}\left(d_{R}\right)\right)^{2}
$$

Many popular criterion minimization methods are based on the computation of the derivative of the criterion. In our case, it would involve the computation of the derivative of $\mathcal{C}$ and consequently of $\partial t / \partial d_{R}$. The computation of the derivative of the depolarization time at a given point with simple finite differences is very tedious and requires two simulation steps which are computationally expensive. More subtle methods like adjoint methods are not directly operative since the depolarization time is not an explicit function of the transmembrane potential. Therefore, we choose a minimization method that does not involve any derivative, an iterative inverse parabolic 
interpolation like the Brent method [28]. This very consistent method replaces the function to be minimized by a well-chosen parabola. The minimum of the function $\mathcal{C}$ is approximated by the easily and efficiently computed minimum of the parabola. Given $\left(d_{a}, \mathcal{C}\left(d_{a}\right)\right),\left(d_{b}, \mathcal{C}\left(d_{b}\right)\right)$ and $\left(d_{c}, \mathcal{C}\left(d_{c}\right)\right)$ three points on the curve, there exists a unique parabola $f(x)=\alpha x^{2}+\beta x+\gamma$ going through these points reaching its extremum at a point $d_{x}$ such that

$$
d_{x}=d_{b}-\frac{1}{2} \frac{\left(d_{b}-d_{a}\right)^{2}\left(\mathcal{C}\left(d_{b}\right)-\mathcal{C}\left(d_{c}\right)\right)-\left(d_{b}-d_{c}\right)^{2}\left(\mathcal{C}\left(d_{b}\right)-\mathcal{C}\left(d_{a}\right)\right)}{\left(d_{b}-d_{a}\right)\left(\mathcal{C}\left(d_{b}\right)-\mathcal{C}\left(d_{c}\right)\right)-\left(d_{b}-d_{c}\right)\left(\mathcal{C}\left(d_{b}\right)-\mathcal{C}\left(d_{a}\right)\right)}
$$

Then $\mathcal{C}\left(d_{x}\right)$ can be computed using a new simulation based upon the model of Aliev and Panfilov with this new value for $d$ and compared with $\mathcal{C}\left(d_{a}\right), \mathcal{C}\left(d_{b}\right)$ and $\mathcal{C}\left(d_{c}\right)$.

We construct an iterative process which is a simplified version of Brent's method [28], to find the minimum from an initial bracketing of the minimum. The bracketing of the minimum of the function $\mathcal{C}$ consists in three points $d_{a}, d_{b}$ and $d_{c}$ such that $d_{a}<d_{b}<d_{c}, \mathcal{C}\left(d_{b}\right)<\mathcal{C}\left(d_{a}\right)$ and $\mathcal{C}\left(d_{b}\right)<\mathcal{C}\left(d_{c}\right)$. We repeat the parabolic estimation until we are satisfied with the obtained value, that is if $\left(d^{k}\right)$ is the sequence of successively estimated minima, we consider that convergence is reached when the difference between two successive estimations is smaller than a given stopping criterion $p$ i.e. $\left|d^{k+1}-d^{k}\right|<p$.

\section{RESULtS}

\section{A. Validation of the methods on simulated data}

We evaluate the performance of the global adjustment of the model on simulated data. The data are simulated on a surface mesh of the epicardium consisting of 192 vertices and 336 triangles. The depolarization time distribution presented on Figure 2 is the result of a simulation based upon the Aliev-Panfilov model for $\varepsilon=0.1, a=0.15, k=12$ and a non constant conductivity map with a mean value of 1.0. The integration time step was chosen $d t=5 \cdot 10^{-4}$ to make the explicit Euler scheme converge. The depolarization time distribution of Figure 2 is now a set of simulated data.

Applying the procedure described in Section II-B to these simulated data, we obtain a global value of $k^{m}=12.51$ starting at a crude initialization of $k^{s}=8$. The values of $a$ and $\varepsilon$ for the crude initialization were chosen equal to the parameters used to simulate the data and the AC $d$ was chosen spatially constant and equal to 1.0. A simulation is then performed with this new $k$ value and the depolarization time distribution is compared with the simulated measures. The depolarization time error drops from $25.3 \mathrm{~ms}$ with $k^{s}=8$ to $14.7 \mathrm{~ms}$ with the new estimated 


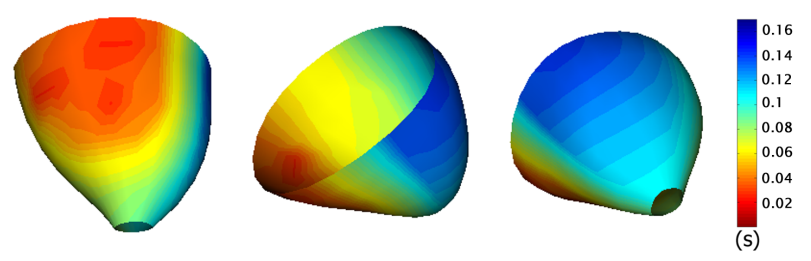

Fig. 2. A surface mesh where the depolarization time is computed for each vertex after simulating the transmembrane potential propagation. The color encodes the depolarization time.

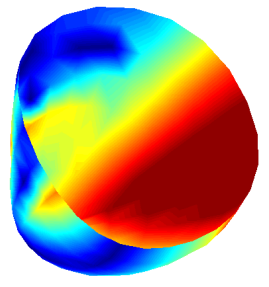

(a)

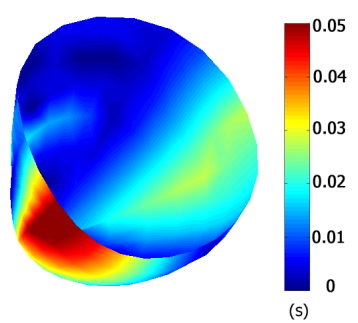

(b)

Fig. 3. Simulated measures: absolute depolarization time error between simulated data and simulations before (a) and after (b) the global automatic estimation.

value $k^{m}=12.51$. Figure 3 displays the error between the measured depolarization time and the simulated depolarization time before and after the estimation of $k$.

We then test the local estimation algorithm on simplified simulated data. We still use the surface mesh of Figure 2. All the parameters but $d$ are fixed: $\varepsilon=0.1, a=0.15, k=12$ and $d t=5.10^{-4}$. We choose only one region $R$ where the $\mathrm{AC}$ value is modified from the standard value 1.0 to a value $d_{R}^{m}$. On the rest of the mesh, the AC is equal to 1 . We perform a new simulation with the modified $\mathrm{AC}$ and produce a map of depolarization time that we consider as input to the $\mathrm{AC}$ estimation algorithm. In this example, we suppose that we know the region $R$ of modified conductivity.

We then present two examples, one with a low conductivity value and one with a large one. In this first example, the expected $\mathrm{AC}$ value is $d_{R}^{m}=0.5$, we choose a very rough initial bracketing, $d_{a}=0.1, d_{b}=2.0$ and $d_{c}=9.0$. We perform the iterative process until the stopping criterion $p$ is lower than $10^{-3} .33$ iterations are necessary to obtain an estimation of $d_{R}=0.501$. Figure 4 (a) shows the evolution of the estimated at each iteration of the minimization. The convergence is fast and stable. 


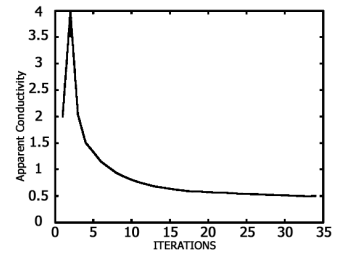

(a)

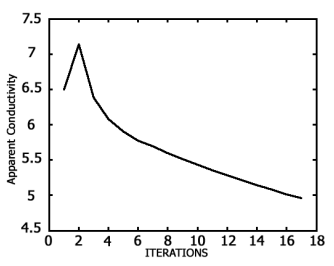

(b)

Fig. 4. Evolution of the estimated apparent conductivity during the estimation. (a): $d_{R}^{m}=0.5, p=10^{-3}$. (b): $d_{R}^{m}=5.0, p=10^{-2}$.

In a second example, the expected $\mathrm{AC}$ value is $d_{R}^{m}=5.0$. We perform the iterative process until the stopping criterion is less than 0.01. By choosing the initial bracketing, $d_{a}=2.0, d_{b}=6.5$ and $d_{c}=9.0$, we assume that the $\mathrm{AC} d_{R}^{m}$ is greater than 2.0. With these parameters, 17 iterations are necessary to reach convergence and obtain $d_{R}=5.02$. As the function $\mathcal{C}\left(d_{R}\right)$ is very flat around its minimum, the convergence is slower with the parameters of the first example, but the estimated $\mathrm{AC}$ is the same. Figure 4(b) shows the iterative estimation of $\mathrm{AC}$ values.

\section{B. Results on in vivo measures. Normal case.}

In this section, we explain in details the procedure for a normal heart. The in vivo measures used in this section were acquired on an adult male mongrel dog using a multi-electrode epicardial sock. The surgery, experimental layout and the data acquisition are described in [26], [19]. This heart was artificially paced and the natural pacing was suppressed. For analysis of electrical activation, epicardial readings from each electrode were averaged over approximately 20 heartbeats. The derivative of the voltage $v(t)$ is computed with a five-point finite difference estimate. The depolarization time is chosen as the instant of the most negative derivative. The depolarization time distribution computed from the potentials recorded on the 128 electrodes of the sock is interpolated on a surface mesh consisting of 336 triangles and 192 vertices as displayed on Figure 5.

The pacing is artificial, consequently it is simple to select the initial pacing area as we show on Figure 5. All the parameters but $k$ are fixed as explain in section II-B, $a=0.15, \varepsilon=0.1, d=1$ and the time step is $d t=5.10^{-4}$. Applying the method presented in Section II-B to the data, we obtain a global value of $k^{m}=25.2$ starting from a crude initialization $k^{s}=8$.

Figure 6(a) presents the depolarization time obtained after the global adjustment. The depolarization time error obtained before and after the automatic global estimation procedure are 


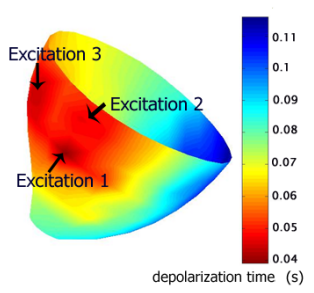

Fig. 5. Measured depolarization time on a normal canine heart with artificial pacing. The arrows indicate the pacing sites selected to initialize the simulations.

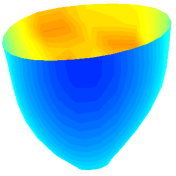

(a)

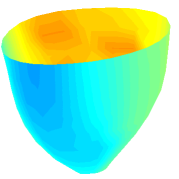

(b)

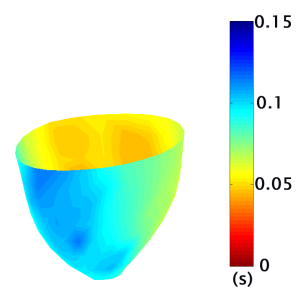

(c)

Fig. 6. Depolarization time before (a) and after (b) the local estimation compared with the measures (c).

shown on Figures 7(a) and 7(b). The depolarization time distribution computed with our procedure (Figure 6(a)) is in the same range of values as the measured depolarization time distribution. This is confirmed by comparing the absolute error between simulated depolarization and measured depolarization time before (Figure $7(\mathrm{a})$ ) and after (Figure 7(b)) the automatic estimation of $k$. Before the estimation, the mean error was $20.6 \mathrm{~ms}$. After the automatic estimation, the mean error is $10 \mathrm{~ms}$.

We now apply the proposed method to perform the local estimation of the AC.

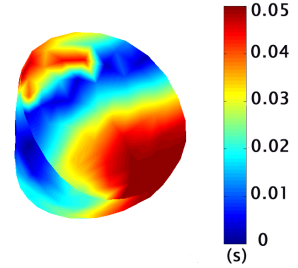

(a)

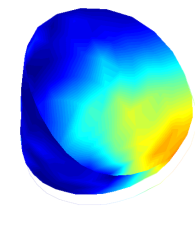

(b)

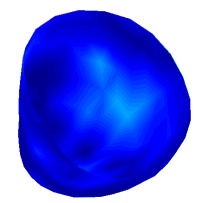

(c)

Fig. 7. Absolute depolarization time error between measures and simulations before (a) and after (b) the global automatic estimation and finally after the local estimation (c) 


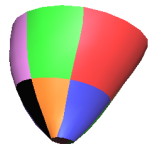

(a)

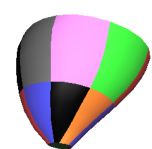

(b)

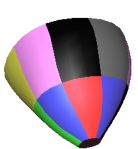

(c)

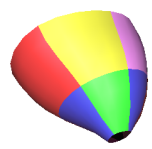

(d)

Fig. 8. The 14 regions chosen on the epicardium, according to the propagation of the depolarization wave. Different views. The large red region contains the pacing electrodes.

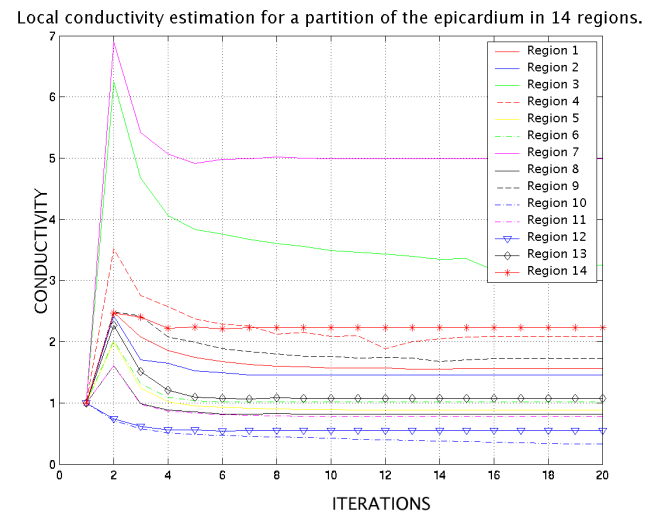

Fig. 9. Convergence of the apparent conductivity for the 14 selected regions.

We first create a partition of the epicardium by splitting the epicardium into regions according to the isochrones of the depolarization time map. The precision of this partition is limited by the mesh resolution. In case this regions are too large we split them orthogonally to the isochrones. Finally, for this example we obtain 14 regions as shown in Figure 8.

We then iteratively estimate one $\mathrm{AC}$ value for each region. We sort the 14 regions of Figure 8 according to their depolarization time. The convergence on each region is presented in Figure 9 while Figure 10 shows different views of the obtained AC map.

The absolute error decreases significantly both after the global estimation and the local
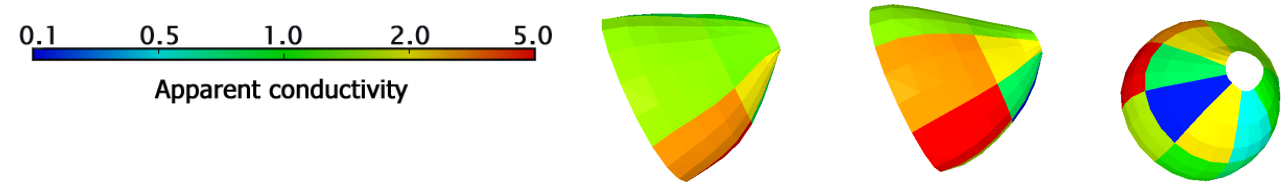

Fig. 10. Apparent conductivity map. Different views. The color represents the apparent conductivity value. From blue (small values) to red (high values). 


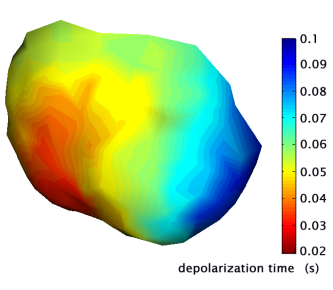

(a)

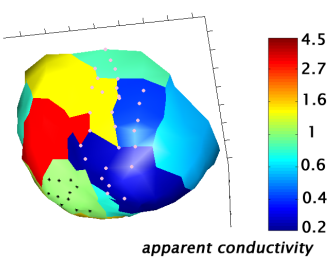

(b)

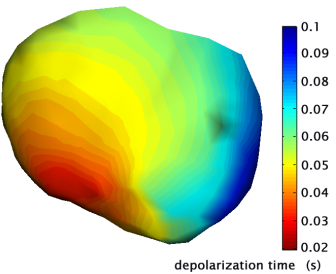

(c)

Fig. 11. Estimation of apparent conductivity for the case of the anterior infarct. (a) Measured depolarization time for an infarcted dog heart. (b) Apparent conductivity estimated. The bright circles indicate the location of the infarct. The points marked with a dark star indicate the pacing sites. The depolarization time distribution computed with these apparent conductivity values is depicted in (c).

estimation. Figure 6 displays the depolarization time simulated by the model before (Figure 6(a)) and after (Figure 6(b)) the local estimation of the AC compared with the measures (Figure 6(c)). Figure 7 displays the absolute error on the depolarization time before and after the local estimation of the AC.

\section{Case of an infarcted heart}

We also apply the AC estimation method on the case of an infarct on the anterior wall. This heart was artificially paced and the measures were obtained from an epicardial sock like in the normal case. The measured depolarization time distribution in this case is depicted in Figure 11(a). The conduction appears to be slower in the infarcted region due to the local modification of the cells properties and of the conductivity. As a consequence, we expect the AC to be low in the infarcted region. The AC values are displayed on Figure 11(b), the bright circles correspond to the approximate location of the infarcted region. The depolarization time distribution computed from a simulation taking into account these values is displayed on Figure 11(c). In the infarcted region, the shape of this depolarization front reproduces the shape of the measured depolarization front (Figure 11(a)).

\section{Discussion}

\section{A. Performances and parameter choice.}

The estimation of the $\mathrm{AC}$ for one region takes about 6 minutes on a dual processor Pentium III $1 \mathrm{GHz}$. The computation time lies mainly in the simulations based on the model. In very 


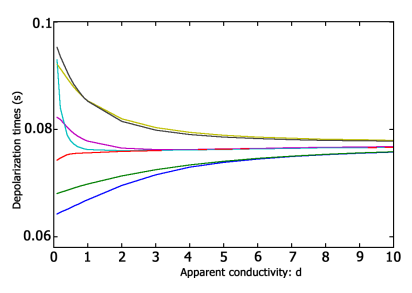

(a)

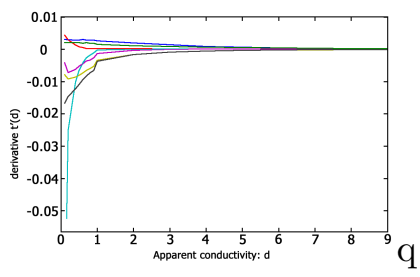

(b)

Fig. 12. (a) Depolarization time as a function of the apparent conductivity $d_{R}$ at the seven vertices of a region $R$. (b) the derivative $\partial t / \partial d$ of these curves

simple simulated examples where precomputed depolarization time can be used, the estimation lasts around 10 seconds.

The convergence of the local estimation algorithm is as stable for the real measures as for the simple synthetic example. The criterion $\mathcal{C}$ is approximated by a parabola, which exposes only one minimum, the global one. Therefore, we do not observe oscillations between several possible local minima. We did not experiment the drawbacks described in [28]. Thus the simplified version of Brent minimization algorithm seems sufficient to minimize $\mathcal{C}(d)$.

This minimization method relies on a few parameters, the initial bracketing and the desired stopping criterion. A difference between two successive iterations lower than 0.01 is sufficient. In a first stage, we consider that no a priori information is available for the AC. A very rough initial bracketing is essential in order to assure that it contains the minimum of $\mathcal{C}$. The $\mathrm{AC}$ should always be positive. Therefore, we take a small value for the lower bracket $d_{a}$. Hence, the positivity of the $\mathrm{AC} d$ is easily imposed. There is no particular maximum limit for the AC. As all the regions belong to the same heart, it is realistic to take an $\mathrm{AC}$ always lower than 10.

An important stage of the estimation is the splitting of the epicardium in several regions where the estimated $\mathrm{AC}$ is constant. For the moment, this partition is tuned manually.

\section{B. Expected accuracy of the apparent conductivity estimation}

Figure 12(a) presents an example of the depolarization time as a function of the $\mathrm{AC} d_{R}$ at the seven vertices of a region $R$ and Figure 12(b) presents the derivative $\partial t / \partial d$ of these curves. When the absolute value of the derivative of $t$ according to $d$ is small, it means that the depolarization time distribution does not change much upon a moderate variation of the $\mathrm{AC}$. The larger is the derivative, the smaller the range of $\mathrm{AC}$ values that leads to approximatively the same depolarization 
time and thus the better estimation of AC. As a consequence, we have here an intrinsic limitation for any $\mathrm{AC}$ estimation process from depolarization time. We also notice that the absolute value of the derivative is greater for small values of $d$ than for large values of $d$. Consequently, we can estimate more accurately small $\mathrm{AC}$ values than larger ones.

\section{Discussion on the results}

The global adjustment is satisfying and provides a good initialization to start the estimation of the local parameter. At this stage, we are more interested in the error on the depolarization time than on the value of $k$. The first reason is that $k$ does not correspond to any measurable quantity that can be extracted from a set of measures. The second reason is that this global estimation is based on a model with spatially constant parameters whereas it may not be the case for simulated or real measures. The error is globally smaller after the estimation of $k$ but the error is still large in regions far from the pacing region. Indeed, the estimation of the parameter $k$ is not performed by controlling directly the depolarization time but by controlling the norm of the spatial gradient of the depolarization time derivative, that is the inverse of the velocity (Section II-B).

The estimated AC values are consistent with the measures. From Figure 9, we visually distinguish two types of regions. The regions of the first type have $\mathrm{AC}$ values around 0.9 and for a second class of regions, the $\mathrm{AC}$ is around 1.8. This reflects the asymmetry of the measures. The depolarization wave is faster on one side than on the other side of the epicardium. This may be due to the fact that we model the epicardium as homogeneous, without distinguishing the left and right ventricle nor taking into account the fiber directions. In agreement with the measures, our estimation algorithm provides us with asymmetric conductivities. On the upper left view of Figure 10, the green region corresponds to the pacing region. On the left, we estimate an AC close to 0.9, whereas on the right we estimate an $\mathrm{AC}$ up to 5.0. If we refer to Equation (3), it means that the velocity of the

propagation is more than twice larger on one side than on the other $(\sqrt{5 / 0.9} \approx 2.34)$.

Let us consider more precisely the first region, which includes the pacing electrodes. In this region, we have a limitation with the global estimation because the depolarization appears to be faster than in other parts of the epicardium. The model with constant parameters is not able to capture this phenomenon. Fortunately with the local estimation, the AC value estimated in this region is greater than 1.0, producing a faster depolarization wave in the pacing region, in perfect agreement with the experiment.

From Figure 6, we notice a visual improvement of the depolarization time distribution. Compar- 
ing these results with the measures (Figure 6(c)), we notice that the shape of the depolarization front resembles much the measures with the local adjustment. The quality of this estimation is also assessed by the visualization of the absolute error on the depolarization time in the epicardial surface. On Figure 7(b), the depolarization time error is small near the pacing region but becomes larger further away from this region. The final depolarization time error (Figure $7(\mathrm{c})$ ) is not only smaller but also more homogeneous. Thus the agreement between the measures and the model is improved in the entire epicardial surface. In conclusion, the error has been considerably improved by the local estimation of the AC, even if we only added 14 degrees of freedom: $14 \mathrm{AC}$ values for 14 regions.

\section{Case with the infarct}

On Figure 11(b), the infarcted region is displayed as bright circles. A large portion of the infarct is detected in the two regions with the lowest conductivity values, but we see that a part of the infarct is not detected as a low conductivity region. The heterogeneous infarct geometry in the heart wall can explain this observation: the infarct can be transmural (i.e. extending from the inner surface to the outer surface) or non-transmural (i.e. extending from the inner surface to somewhere in the wall), and when considering vertices in the mesh, where the infarct is non-transmural, electrical conductivity can be almost normal. In addition, a low conductivity is estimated in normal regions. As seen in the first case, this may be due to the modeling of the epicardium as homogeneous medium. We are currently working on the inclusion of the fiber directions in this model.

\section{Conclusions And Perspectives}

We addressed the problem of estimating a set of parameters for the transmembrane potential propagation modeled by Aliev and Panfilov from measured depolarization time. In order to evaluate the quality of our results, we used a criterion based on the difference of depolarization time between the model and the measures. We first presented a procedure to estimate globally a set of parameters so as to properly scale the electrical propagation. We used the theoretical properties of the Aliev and Panfilov equations and validated that approach on simulated depolarization time. We then presented a method to locally estimate the apparent conductivity (AC) region by region. This method was validated on simple simulated depolarization time. We successfully estimated global and local parameters of the model from in vivo measures of a canine heart. The simulation based on the model with these new values showed that the depolarization time error was significantly 
decreased. Moreover, the $\mathrm{AC}$ values that we obtained are consistent with the measures even if a ground truth is not available. We also applied the estimation of the local AC to the case of an infarcted heart and found a strong correlation between parts of low AC values and the infarcted region.

In order to have a totally automatic process, we still have to find and build algorithms for the automatic partitioning of the epicardium. The proposed method could be easily extended to include more input data, like the isochrones of repolarization time, thus leading to the estimation of one additional spatially varying parameter (in this case the $a$ parameter). In other words, we presented a general framework that is applicable to one or more macroscopic observations of cardiac electrophysiology. The model based approach that we propose could also be extended to a 3D model and could easily take into account fiber directions. As an isotropic model is too limited, we first plan to introduce anisotropy in the 2D model. The next step will be to estimate the parameters of a 3D heart model by establishing a correspondence between 2D measures and a 3D mesh. The proposed approach is not dependent on the model since it only uses simulations based on the forward model. Thus, it can be adapted to more complex models that can reproduce specific pathologies. It can also be adapted to models computing depolarization time rather than transmembrane potential like eikonal models [29]. Finally a truly physiological validation would require to apply our method to a benchmark of pathological and normal measures analyzed by experts.

The electrophysiological measures used in this article are very rich but also very invasive. Therefore, a long term challenge will be to estimate parameters of a full electromechanical model using both ECGs and displacement data, for example tagged MRI [30]. This problem is more complex because the equations modeling the mechanical deformation are more unstable than for the electrophysiological models. First results concerning the estimation of mechanical parameters from MR images were obtained recently in [31].

\section{ACKNOWLEDGMENTS}

This work was partially funded by the scientific direction of INRIA through the Cooperative Research Action ICEMA2 (http://www-rocq.inria.fr/sosso/icema2/icema2.html). This action involved several partners including the INRIA research groups CAIMAN, EPIDAURE, MACS and SOSSO and was coordinated by Frédérique Clément (SOSSO). 


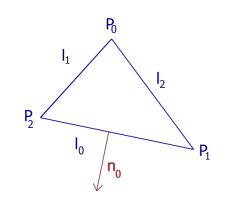

Fig. 13. Notations used on a triangle $\left(\mathbf{P}_{0} \mathbf{P}_{1} \mathbf{P}_{2}\right)$.

\section{APPENDIX}

On each triangle trngl $=\left(\mathbf{P}_{0} \mathbf{P}_{1} \mathbf{P}_{2}\right)$ of the triangulation, the gradient of $t$ is

$$
\boldsymbol{\nabla}_{\mathbf{x}} t(\text { trngl })=-\sum_{i=0}^{2} \frac{\mathbf{S}_{\mathbf{i}}}{2 \mathcal{A}} t\left(\mathbf{P}_{i}\right)
$$

where $\mathcal{A}$ is the area of the triangle $\left(\mathbf{P}_{0} \mathbf{P}_{1} \mathbf{P}_{2}\right)$ and $\mathbf{S}_{\mathbf{i}}=l_{i} \mathbf{n}_{\mathbf{i}}, l_{i}$ is the length of the segment $\mathbf{P}_{i+1} \mathbf{P}_{i+2}$ and $\mathbf{n}_{\mathbf{i}}$ is the external normal to this segment (Figure 13). We denote $\mathcal{S H}(v)$ the shell of the vertex $v$, that is the set of triangles to which $v$ belongs. Then for each vertex $v \in \mathcal{V}$, the gradient $\nabla_{\mathbf{x}} t$ is given by

$$
\nabla_{\mathbf{x}} t(v)=\frac{\sum_{\text {trngl } \in \mathcal{S H}(v)} \nabla_{\mathbf{x}} t(\text { trngl }) \mathcal{A}(\text { trngl })}{\sum_{\text {trngl } \in \mathcal{S H}(v)} \mathcal{A}(\text { trngl })}
$$

where $\mathcal{A}$ (trngl) is the area of triangle trngl.

\section{REFERENCES}

[1] Y. Rudy, "Inverse computation of epicardial potentials," International Journal of Bioelectromagnetism, vol. 5, no. 1, pp. 218-220, 2003.

[2] A. McCulloch, D. Sung, M. Thomas, and A. Michailova, "Experimental and computational modeling of cardiac electromechanical coupling," in Functional Imaging and Modeling of the Heart (FIMH), ser. LNCS, no. 2230. Springer, 2001, pp. 113-119.

[3] O. Faris, F. Evans, D. Ennis, P. Helm, J. Taylor, A. Chesnik, M. Guttman, C. Ozturk, and E. McVeigh, "A novel technique for cardiac electromechanical mapping with MRI tagging and an epicardial electrode sock," Annals of Biomedical Engineering, vol. 31, no. 4, pp. 430-440, 2003.

[4] K. Rhode, M. Sermesant, G. Sanchez-Ortiz, S. Hegde, D. Rueckert, D. Hill, and R. Razavi, "XMR guided cardiac electrophysiology study and radio frequency ablation," in SPIE Medical Imaging, A. Amini, Ed., 2004.

[5] D. Noble and Y. Rudy, "Models of cardiac ventricular action potentials: iterative interaction between experiment and simulation." Phil. Trans. R. Soc. Lond. A, pp. 1127-1142, 2001.

[6] D. Noble, "Modeling the heart from genes to cells to the whole organ," Science, vol. 295, pp. 1678-1682, march 2002.

[7] M. Belik and A. McCulloch, "Computational methods for cardiac electrophysiology," in Computational Models for the Human Body, ser. Handbook of Numerical Analysis, N. Ayache, Ed. Elsevier, 2004. 
[8] V. Krinski, A. Pumir, and I. Efimov, "Cardiac muscle models," in Encyclop. of Nonlin. Science, A. Scott, Ed. Routledge, 2004.

[9] P. Hunter, "The IUPS physiome project: a framework for computational physiology," Progress in Biophysics and Molecular Biology, vol. 85, no. 2-3, pp. 551-569, 2004.

[10] C. Luo and Y. Rudy, "A model of the ventricular cardiac action potential. Depolarization, repolarization and their interaction." Circulation Research, vol. 68, no. 6, pp. 1501-1526, 1991.

[11] — "A dynamic model of the cardiac ventricular action potential. simulations of ionic currents and concentration changes," Circulation Research, vol. 74, no. 6, pp. 1071-1097, 1994.

[12] R. FitzHugh, "Impulses and physiological states in theoretical models of nerve membrane," Biophysical Journal, vol. 1 , pp. $445-466,1961$.

[13] F. Fenton and A. Karma, "Vortex dynamics in three-dimensional continuous myocardium with fiber rotationfilament instability and fibrillation." Chaos, vol. 8, pp. 20-47, 1998.

[14] O. Bernus, R. Wilders, C. Zemlin, H. Verschelde, and A. Panfilov, "A computationnally efficient electrophysiological model of human ventricle cells." American Journal of Physiology Heart and Circulation Physiology, vol. 282, no. 6, pp. 2296-2308, 2002.

[15] K. T. Tusscher, D. Noble, P. J. Noble, and A. V. Panfilov, "A model for human ventricular tissue," American Journal of Physiology Heart and Circulation Physiology, vol. 286, no. 4, pp. 1573-1589, 2003.

[16] P. Colli-Franzone, L. Pavarino, and B. Taccardi, "Monodomain simulations of excitation and recovery in cardiac blocks with intramural heterogeneity," in FIMH, ser. LNCS, no. 3504. Springer, 2005, pp. 267-277.

[17] R. Clayton and A. Holden, "Propagation of normal beats and re-entry in a computational model of ventricular cardiac tissue with regional differences in action potential shape and duration," Progress in Biophysics and Molecular Biology, vol. 85, no. 2-3, pp. 473-499, 2004.

[18] R. Aliev and A. Panfilov, "A simple two-variables model of cardiac excitation," Chaos, Soliton and Fractals, vol. 7, no. 3, pp. 293-301, 1996.

[19] M. Sermesant, O. Faris, F. Evans, E. McVeigh, Y. Coudière, H. Delingette, and N. Ayache, "Preliminary validation using in vivo measures of a macroscopic electrical model of the heart," in Int. Symp. on Surgery Simulation and Soft Tissue Modeling (IS4TM), vol. 2673. Springer-Verlag, 2003, pp. 230-243.

[20] R. van der Merwe and E. Wan, "The square root unscented Kalman filter for state and parameters estimation," in Int. Conf. on Acoustics, Speech and Sign. Process. (ICASSP), May 2001.

[21] G. Evensen, D. Dee, and J. Schröter, "Parameter estimation in dynamical models," in Ocean Modelling and Parameterization, NATO ASI, E. J.Verron and G.Chassignet, Eds., 1998.

[22] P. Shi and H. Liu, "Stochastic finite element framework for cardiac kinematics function and material property analysis," in Medical Image Computing and Computer-Assisted Intervention (MICCAI), ser. LNCS, vol. 2488. Springer, September 2002, pp. 635-641.

[23] N. Ayache, D. Chapelle, F. Clément, Y. Coudière, H. Delingette, J. Désidéri, M. Sermesant, M. Sorine, and J. Urquiza, "Towards model-based estimation of the cardiac electro-mechanical activity from ECG signals and ultrasound images," in FIMH, ser. LNCS, no. 2230. Springer, 2001, pp. 120-127.

[24] M. Sermesant, C. Forest, X. Pennec, H. Delingette, and N. Ayache, "Biomechanical model construction from different modalities: Application to cardiac images," in MICCAI, ser. LNCS, vol. 2488. Springer, 2002, pp. $714-721$. 
[25] D. Chapelle, F. Clément, F. Génot, P. L. Tallec, M. Sorine, and J. Urquiza, "A physiologically-based model for the active cardiac muscle contraction," in FIMH, ser. LNCS, no. 2230. Springer, 2001, pp. 128-133.

[26] V. Moreau-Villéger, H. Delingette, M. Sermesant, O. Faris, E. McVeigh, and N. Ayache, "Global and local parameter estimation of a model of the electrical activity of the heart," INRIA, Research Report 5269, 2004.

[27] J. Keener, "A geometrical theory for spiral waves in excitable media," SIAM Journal in Applied Mathematics, vol. 46, no. 6, pp. 1039-1056, 1986.

[28] W. Press, B. Flannery, S. Teukolsky, and W. Vetterling, Numerical Recipes in C. Cambridge University Press, 1991, ch. Minimization or Maximization of Functions, pp. 290-352.

[29] P. Colli-Franzone and L. Guerri, "Spreading of excitation in 3d models of anisotropic cardiac tissue :i. validation of the eikonal model," Mathematical Bioscience, vol. 113, pp. 145-209, 1993.

[30] L. Axel, A. Montillo, and D. Kim, "Tagged magnetic resonance imaging of the heart: a survey," Medical Image Analysis, vol. 9, no. 4, pp. 376-393, 2005.

[31] M. Sermesant, P. Moireau, O. Camara, J. Sainte-Marie, R. Andriantsimiavona, R. Cimrman, D. Hill, D. Chapelle, and R. Razavi, "Cardiac function estimation from MRI using a heart model and data assimilation: Advances and difficulties," in FIMH, ser. LNCS, no. 3504. Springer, 2005, pp. 325-337. 